\title{
MICROENCAPSULATION OF TRIOCTYLAMINE IN POLYMERIC MATRICES FOR REMOVING Zn(II) AND Cu(II) FROM CHLORIDE AQUEOUS SOLUTIONS
}

\author{
C. FONSECA ${ }^{A}$, C. ARANEDA $A^{A}$, M. YAZDANI-PEDRAM ${ }^{B}$, T. BORRMANN ${ }^{C}$, C. BASUALTO ${ }^{4}$, \\ J. SAPAGA AND F. VALENZUELA $A^{A^{*}}$ \\ ${ }^{a}$ Laboratorio de Operaciones Unitarias e Hidrometalurgia, Facultad de Ciencias Químicas y Farmacéuticas, Universidad de Chile, \\ PO Box Santiago 1, 233, Chile. \\ ${ }^{b}$ Laboratorio Quimica de Polimeros, Facultad de Ciencias Quimicas y Farmacéuticas, Universidad de Chile, PO Box Santiago 1, 233, Chile \\ ${ }^{c}$ School of Chemical and Physical Science, Victoria University of Wellington, \\ New Zealand. PO Box 600, Wellington, New Zealand. \\ (Received: May 5, 2010 - Accepted: July 15, 2010)
}

\begin{abstract}
The microencapsulation of the non-specific basic extractant trioctylamine in polymeric matrices synthesized from styrene and divinylbenzene was studied. The microcapsules were prepared by adding the amine compound during in situ free radical suspension polymerization using benzoyl peroxide as the initiator and using variable proportions of both monomers. SEM analysis shows that the microcapsules have a spherical shape presenting an average surface area of 480 $\mathrm{m}^{2} \mathrm{~g}^{-1}$ and a pore size around 0.5 to $1.8 \mathrm{~nm}$. Synthesis of the microspheres was affected by the amount of extractant used and by the proportion of both monomers during their preparation.

The obtained microcapsules were used for the sorptive removal of $\mathrm{Zn}(\mathrm{II})$ and $\mathrm{Cu}(\mathrm{II})$ ions from chloride aqueous solutions, reaching extraction extents near $90 \%$ under the best conditions, following an anion-exchange mechanism between the metallic ions and the extractant immobilized onto the microcapsules. Zn(II) and $\mathrm{Cu}(\mathrm{II})$ sorption kinetics experiments were performed and efficient uptake of both metals within a few minutes was measured. The experimental results were explained using a pseudo-second-order rate kinetics model, which fit the results of chemisorption of both metals onto the microcapsules well.
\end{abstract}

Keywords: Microcapsules, Trioctylamine, Metals, Adsorption, Wastewater treatment

\section{INTRODUCTION}

Many industrial processing systems generate polluted wastewaters containing chloride ions, as well as many other metallic ions, which have to be removed previous to their discharge to surface water bodies or even to municipal sewer systems. Examples of this situation are found in many industrial processes involving plating, tanning, pickling, seafood canning, the manufacture of dyes and ceramic materials, and those industrial processes using ferric chloride as oxidant or as flocculant for removing colloidal materials. In mining, metal extraction processes in a chloride medium are used extensively; for instance, for removal of copper from molybdenite concentrate $\left(\mathrm{MoS}_{2}\right)$ using $\mathrm{FeCl}_{3}$ concentrate solutions as powerful oxidant leachant agents, for the recovery processing of titanium from rutile $\left(\mathrm{TiO}_{2}\right)$ or ilmenite $(\mathrm{FeTiO})$ ores using chloride gas to produce titanium tetrachloride in a preparative step for its final reduction to metal ${ }^{1}$, or for the Cyprus copper and CLEAR refractorycopper sulfide leaching processes using acidified cupric chloride-bearing brine solutions ${ }^{2}$. In all these industrial processes, residual solutions in a chloride medium are created that contain variable amounts of ferrous and non-ferrous metals, among them $\mathrm{Cu}(\mathrm{II}), \mathrm{Zn}(\mathrm{II})$ and $\mathrm{Cd}(\mathrm{II})$, all with concentrations above allowable environmental discharge limits. Additionally, these solutions are typically acidic, which obligates the use of a treatment to remove or recover the metal contents prior to recycling the high-chloride concentration solutions back to the production circuits.

Most of the heavy metals found in these waste solutions pose severe toxicological effects on aquatic ecosystems since they can be adsorbed on plants and fish. Long-term exposure to even low concentrations of metals can cause disease or mortality in fish and other aquatic organisms. On the other hand, some of these metal ions also contain economic value which is motivation itself for finding simpler and more economic methodologies to uptake the metallic ions existent in these waste solutions.

Many technological alternatives can be employed to extract metals from aqueous solutions, however, all of them present some advantages but also multiple limitations, including in some cases a restrictively high-cost to apply them. For example, the use of solid ion-exchange resins (IX process) is limited to low concentrations of metals in solutions and the resins are expensive and are not always selective because their regeneration after saturation is slow and troublesome ${ }^{3}$. Chemical precipitation requires a large quantity of chemical reagents, which generate secondary pollutants, and produces a huge volume of sludge difficult to separate and dispose of in a safe way ${ }^{4}$. Furthermore, several amphoteric precipitates are unstable and tend to re-dissolve according to the acidity of the wastewater. Solvent extraction (SX process) could be an interesting alternative; however, it is more of a productive process to recover metals than a technique to treat polluted wastewaters, although in some cases has been applied for this purpose ${ }^{5}$. However SX is not free of problems, such as the need for using large quantities of expensive organic solvents and the presence of operational difficulties like the loss of the solvent and extractant from the organic phase because of their volatility, mechanical entrainment to the aqueous phase during stirring and crud formation from the fine solid particles in the feed solution to treat.

A method to overcome these disadvantages is to immobilize the liquid organic extractant in a hydrophobic porous polymeric matrix which acts as a suitable support ${ }^{6-8}$. Specifically, we have worked over the last few years on the synthesis and application of microcapsules (MC) containing diverse extractant compounds, which have been prepared in situ by a polymerization method 9,10 . Different microspheres have been synthesized using various monomers and extractant molecules. These microcapsules can be employed in batchwise reactors or in continuous columns. As has been reported in previous works, microcapsules present a large specific interfacial area per volume unit, a high selectivity to the selected extractant to immobilize in the polymeric matrix, and require a minimal inventory of organic solvents for the extraction of the same amount of metal in comparison with SX processing. Additionally, microcapsules are easily separated from the solution by conventional filtration or sedimentation, becoming a convenient alternative to be used in separation processes with respect to current technologies, especially for treating dilute aqueous solutions as many industrial wastewaters.

In recent years the microencapsulation of extractants has been studied in hydrometallurgy for the removal or recovery of various metallic ions from different types of aqueous solutions. Kamio et al. have published several papers concerning the use of microcapsules containing organophosphorous as the active extractant for metals like Ga(III), In(III) and some rare earths, confirming the advantages of the use of microcapsules, including their stability attributes and metal loading-capacities ${ }^{11}$. In other work, Nishihama et al. studied the separation and recovery of $\mathrm{Cr}(\mathrm{VI})$ from simulated plating waste using microcapsules containing a quaternary ammonium salt and a phosphoric acid as extractants ${ }^{12}$. The same methodology was used by Shiomori et al. in an extraction equilibrium study of precious metals from aqueous acidic solutions using microspheres constituted by a divinylbenzene polymeric matrix and a ternary amine-type extractant ${ }^{13}$.

In a previous report, we studied the removal of $\mathrm{Cd}(\mathrm{II})$ and $\mathrm{Cu}(\mathrm{II})$ from aqueous solutions by sorption onto microcapsules prepared by copolymerizing 
ethylene glycol dimethacrylate and styrene ${ }^{14}$. The microcapsules were prepared by an in situ radical polymerization method by adding the extractant compound, 2-ethylhexy phosphonic acid mono-2-ethylhexyl ester, and the monomers to the continuous aqueous phase. A pseudo-second-order kinetics model was applied to the experimental data of the chemisorption of metals onto the microcapsules, which fitted the experimental observations well. Recently, we have published a study concerning the adsorption of $\mathrm{Cr}(\mathrm{VI})$ ions from an aqueous acid solution on hydrophobic polymeric microcapsules containing a long-chain quaternary ammonium salt-type extractant immobilized in their pore structure ${ }^{15}$. It was found that Langmuir's isotherms correlated well with the experimentally observed sorption data. The results of the kinetics experiments, carried out at different temperatures, confirmed that the sorption process is controlled by a chemisorption mechanism with a measured activation energy of $82.7 \mathrm{~kJ} \mathrm{~mol}^{-1}$. The calculation of thermodynamic parameters (change in standard free energy, enthalpy, and entropy) were also made, indicating that the chemisorption process is spontaneous, exothermic and also a less chaotic system than a liquid-liquid extraction system.

In this work, the extraction of $\mathrm{Zn}(\mathrm{II})$ and $\mathrm{Cu}(\mathrm{II})$ using microcapsules containing the compound, trioctylamine (TOA), have been studied. Amines have been used as an effective extractants in liquid-liquid extraction processes (SX) for both metals studied in this work ${ }^{16}$. The microcapsules were prepared by copolymerization of styrene and divinylbenzene using a free radical suspension method. The synthesis and characterization of MC were studied, as well as the metallurgical sorption capacity of both metals from an aqueous solution with a high level of chloride ions that simulates mining wastewater similar to the examples given above. Kinetics information obtained from these experimental results is analyzed and discussed. The basic information deduced from this study will contribute to the design of a pilot-scale continuous column that is loaded with active microcapsules for treating actual wastewaters.

\section{EXPERIMENTAL}

\subsection{Reagents and Solutions}

Styrene $\left(\mathrm{C}_{8} \mathrm{H}_{8}\right.$, molecular weight $\left.104.15 \mathrm{~g} \mathrm{~mol}^{-1}\right)$ and divinylbenzene $\left(\mathrm{C}_{10} \mathrm{H}_{10}\right.$, molecular weight 130.19$)$ supplied by ALDRICH were used as monomers to prepare the microcapsules. The divinylbenzene contains $20 \%$ $\mathrm{w} / \mathrm{w}$ of styrene according to the producer and that value is used to determine the composition of both monomers in the experiments of this study. Reagent grade benzoyl peroxide was used as polymerization initiator. Trioctylamine $\left(\mathrm{C}_{24} \mathrm{H}_{51} \mathrm{~N}\right.$, molecular weight 353.68$)$ supplied by Merck was used without further purification as an extractant. This tertiary amine is a basic extractant that acts by anion exchange with a specific gravity of $0.81\left(20^{\circ} \mathrm{C}\right)$. Toluene was employed as solvent for organic compounds and arabic gum as a dispersant during the preparation of the microcapsules; both were of reagent grade. In addition, analytical grade sodium carbonate $\left(\mathrm{Na}_{2} \mathrm{CO}_{3}\right.$, molecular weight 106.0) and perchloric acid $\left(\mathrm{HClO}_{4}\right.$, molecular weight 100.46) were used for the titration of the extractant immobilized in the microcapsules.

Feed aqueous solutions that simulate mining wastewater with a highchloride content were prepared by dissolving $\mathrm{CuCl}_{2} \cdot 2 \mathrm{H}_{2} \mathrm{O}$ (molecular weight 170.48) and $\mathrm{ZnCl}_{2}$ (molecular weight 136.28). Their initial $\mathrm{pH}$ was adjusted to around a value of 2.0 using hydrochloric acid and was measured using a $\mathrm{pH}$ meter (Oakton). When necessary, sodium chloride (Merck r.g.) was also added to the feed solutions to adjust the chloride concentration.

\subsection{Microencapsulation of trioctylamine (TOA)}

The synthesis of microcapsules was carried out in a batch-type reactor initially containing distilled water, $\mathrm{HCl}$ and arabic gum that constituted a continuous aqueous phase, which was continually agitated using a mechanical stirrer. A small molar-excess of $\mathrm{HCl}$ acid with respect to the amount of amine was used to form the amine salt during the synthesis. In each experiment $4.5 \mathrm{~g}$ of the arabic gum were used as a protective colloid that aids the stabilization and dispersal of small drops formed during the stirring. Next, when a temperature of $75^{\circ} \mathrm{C}$ was reached, an organic solution of the monomers, the polymerization initiator and the extractant dissolved in toluene was added and dispersed in the continuous aqueous phase. Different microcapsules were prepared varying the ratio of the styrene (ST) and divinylbenzene (DVB) monomers. The polymerization was achieved by heating and mixing both phases at $75^{\circ} \mathrm{C}$ for 3 hrs under an inert atmosphere $\left(\mathrm{N}_{2}\right)$ to bring about the conversion of monomers to a polymeric matrix containing the liquid extractant inside their porous structures. The obtained microcapsules were filtered and repeatedly washed with distilled water and then air-dried overnight at room temperature.

\subsection{Characterization of microcapsules}

The content of trioctylamine retained in the microspheres was determined by potentiometric titration in an Oakton $\mathrm{pH} 500$ series $\mathrm{pH}$ meter. In this procedure $1.0 \mathrm{~g}$ of microcapsules was mixed for one hour with $50 \mathrm{~mL}$ of a solution of $\mathrm{Na}_{2} \mathrm{CO}_{3}$ in a PolyScience orbital shaker in order to the regenerate the amine before titration. Then the microcapsules were separated from the basic solution by filtration. Next, the microspheres were suspended in toluene and acetic anhydride and titrated with $\mathrm{HClO}_{4} 0.01 \mathrm{~N}$.

The synthesized microcapsules containing the tertiary amine were observed by scanning electron microscopy (SEM) using a field emission apparatus Jeol, JSM 6500F according a current procedure that involves coating the samples with platinum prior to measurements to suppress the surface charging effect.

The surface area, the pore volume and the pore size of the microcapsules were determined by porosimetry using a gaseous $\mathrm{N}_{2}$ sorptometer in a Micromeritics ASAP 2010 porosimeter. The particle sizes of the polymeric sorbents were measured using Malvern Mastersizer Hydro 2000 MU apparatus. Samples were dispersed in water and ultrasonicated prior to measurement.

\subsection{Metal adsorption kinetics experiments}

In order to examine the kinetics behavior of the microcapsules during the adsorption of metals onto their surfaces, several extraction experiments were done according to the experimental plan. Adsorption kinetics experiments of $\mathrm{Zn}$ (II) and $\mathrm{Cu}$ (II) were carried out in a batchwise reactor by combining $0.5 \mathrm{~g}$ of microcapsules containing the amine extractant and $100 \mathrm{~mL}$ of the aqueous solutions containing $100 \mathrm{mg} \mathrm{L}^{-1}$ of either zinc or copper ions in a flask continuously stirred using a turbine impeller. In addition to their metals content, aqueous solutions also contained $100 \mathrm{~g} \mathrm{~L}^{-1}$ of sodium chloride to ensure the formation of copper and zinc chloride complexes. The $\mathrm{pH}$ of feed solutions was adjusted to a value of 2.0 with hydrochloric acid. All these experimental conditions were chosen trying to simulate the features of a typical polluted wastewater.

The kinetics experiments were carried out, at a controlled temperature of $30^{\circ} \mathrm{C}$, by measuring the variation of the metal concentration in the feed solution from 1 to 120 minutes. In all experiments, once the time had lapsed, the microcapsules were separated from the suspension using a Millipore filter, and the metal concentration in the resulting aqueous solution (raffinate) was determined by atomic absorption spectrophotometry on a GBC 906 AA apparatus. The quantity of metal adsorbed onto the microcapsules was estimated from the mass balance between the initial metal concentration in the aqueous solution and that measured in the raffinates.

\section{RESULTS AND DISCUSSION}

\subsection{Synthesis of microcapsules}

The microcapsules were synthesized using different ratios of styrene and divinylbenzene to form the polymeric matrix. Table 1 shows the mass of each monomer used in the MC preparation, the amount of the tertiary amine added as the extractant and the quantity of benzoyl peroxide employed as the polymerization initiator. Moreover, the table includes the percent yield of the microcapsules that was obtained in each experiment. In order to identify each microcapsule type, they are named by indicating in the subscript the ratio of styrene to divinylbenzene, followed by the weight percentage of extractant added during synthesis; e.g., $\mathrm{MC}_{40 / 60} 19.6$ indicates microcapsules comprised of $40 \%$ styrene and $60 \%$ 
Table 1: Preparation of microcapsules in function of monomer and extractant composition.

\begin{tabular}{|c|c|c|c|c|c|c|}
\hline $\mathrm{MC}_{\text {ST/DVB }}$ TOA & $\begin{array}{c}\text { Styrene } \\
{[\mathrm{g}]}\end{array}$ & $\begin{array}{c}\text { Divinylbenzene } \\
{[\mathrm{g}]}\end{array}$ & $\begin{array}{c}\text { Trioctylamine } \\
{[\mathrm{g}]}\end{array}$ & $\begin{array}{c}\text { Benzoyl Peroxide } \\
{[\mathrm{g}]}\end{array}$ & $\begin{array}{c}\text { Total weight } \\
{[\mathrm{g}]}\end{array}$ & Yield [\%] \\
\hline $\mathrm{MC}_{20 / 80} 4.4$ & 0.914 & 3.656 & 0.25 & 0.930 & 5.75 & 82.09 \\
\hline $\mathrm{MC}_{20 / 80} 6.6$ & 0.900 & 3.600 & 0.38 & 0.910 & 5.79 & 65.46 \\
\hline $\mathrm{MC}_{20 / 80} 8.4$ & 0.914 & 3.656 & 0.50 & 0.910 & 5.98 & 66.72 \\
\hline $\mathrm{MC}_{20 / 80} 11.0$ & 0.912 & 3.648 & 0.68 & 0.970 & 6.21 & 62.00 \\
\hline $\mathrm{MC}_{20 / 80} 12.1$ & 0.908 & 3.632 & 0.75 & 0.920 & 6.21 & 56.68 \\
\hline $\mathrm{MC}_{20 / 80} 14.7$ & 0.908 & 3.632 & 0.95 & 0.970 & 6.46 & 48.61 \\
\hline $\mathrm{MC}_{40 / 60} 19.6$ & 1.704 & 2.776 & 1.32 & 0.920 & 6.72 & 29.02 \\
\hline $\mathrm{MC}_{40 / 60} 23.0$ & 1.642 & 2.888 & 1.64 & 0.950 & 7.12 & 23.60 \\
\hline $\mathrm{MC}_{40 / 60} 26.2$ & 1.806 & 2.824 & 1.99 & 0.970 & 7.59 & 35.70 \\
\hline $\mathrm{MC}_{40 / 60} 31.7$ & 1.696 & 2.744 & 2.48 & 0.900 & 7.82 & 36.57 \\
\hline $\mathrm{MC}_{40 / 60} 36.1$ & 1.706 & 2.864 & 3.10 & 0.930 & 8.60 & $\mathrm{NRO}$ \\
\hline $\mathrm{MC}_{60 / 40} 22.7$ & 2.774 & 1.860 & 1.62 & 0.900 & 7.15 & 41.12 \\
\hline $\mathrm{MC}_{60 / 40} 29.4$ & 2.678 & 1.830 & 2.26 & 0.930 & 7.70 & 55.84 \\
\hline $\mathrm{MC}_{60 / 40} 34.1$ & 2.694 & 1.820 & 2.81 & 0.920 & 8.24 & 49.51 \\
\hline $\mathrm{MC}_{60 / 40} 36.2$ & 2.810 & 1.800 & 3.15 & 0.930 & 8.69 & 38.90 \\
\hline
\end{tabular}

NRO: No Reaction Observed

divinylbenzene and containing $19.6 \% \mathrm{w} / \mathrm{w}$ of TOA, which in this case refers to the mass of the amine over the total mass of organic compounds used in the preparation.

Three series of microcapsules were synthesized containing $20 \%$ of styrene and $80 \%$ of divinylbenzene, $40 \%$ of styrene and $60 \%$ of divinylbenzene and $60 \%$ of styrene and $40 \%$ of divinylbenzene. As can be observed in Table 1 , the yield of microcapsules obtained varied widely, from $23.6 \%$ to $82.1 \%$. It is clear that a larger amount of trioctylamine added in the polymerization reaction can cause an inhibition of the synthesis of the microspheres. In some cases, the reaction did not even take place or either the quality of the microcapsules was not appropriate; one possible reason being that under these conditions, the free radicals generated by the thermal decomposition of benzoyl peroxide would be deactivated by collisions with extractant molecules. The higher the quantity of TOA in the polymerization process, the higher the probability of deactivation of the free radicals of the initiator and of the intermediate compounds, resulting in a lower microcapsule yield. Furthermore, too high of a concentration of the extractant during the preparation would produce - as it has been measured in liquid-liquid extraction experiments - an excessive increase in the viscosity of the organic phase in the reactant mix, affecting the diffusion of species in the reaction system, which also would provoke a drop in the yield of the synthesized microcapsule.

The role of DVB on the synthesis and stability of MC is important. The divinylbenzene monomer produces a branched polymer structure, like a porous three-dimensional net, acting as an efficient cross-linking agent. Thus, a flexible polymeric matrix is formed that is able to retain the extractant and the metalextractant complex because of its hydrophobic character while also allowing the necessary diffusion of species through the matrix. As has been reported in previous works ${ }^{14,15}$, the sorption of metals onto the microcapsules would follow a mechanism that describes the diffusion of metal within the aqueous phase film that is close to the MC surface, the chemical reaction of the extractant with the metal in the surface of the microcapsule and the intraparticle diffusion of the metal-complex within the porous structure of the MC. Thus, if a too rigidly structured microcapsule was synthesized, its capacity as an adsorbent would be affected. In fact, no leakage of extractant from the microcapsules was observed under the experimental conditions used in this study, confirming that the hydrophobicity of the amine extractant matches the nature of the polymeric matrix. However, the styrene monomer, although less hydrophobic than divinylbenzene, imparts a convenient hydrophilic-hydrophobic equilibrium that assures the wettability of the microcapsules, allowing the chemisorption of the metallic ions present in the feed solutions onto the whole structure of the microsphere. Then, a polymeric matrix prepared with both monomers assure a stable and active microcapsule.

\subsection{Characterization of microcapsules}

Figure 1 shows the scanning electron microscope (SEM) micrographs of one of the synthesized microcapsules containing trioctylamine.
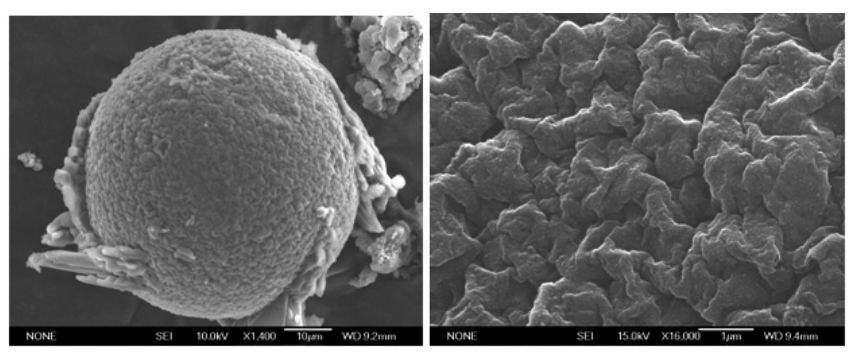

Figure 1: SEM micrographs of microcapsule $\mathrm{MC}_{60 / 40}$ 34.1: (a) whole image of microsphere, (b) surface structure.

Figure 1(a) shows the entire image of $\mathrm{MC} \mathrm{MC}_{60 / 40} 34.1$, and Figure 1(b) a view of its surface. All the microcapsules have a spherical shape and a rather rough surface, including many corrugations on its external surface, which increases the surface area. That is a beneficial quality because a higher surface area allows for better mass transfer of metal from the aqueous solution towards the MC by enhancing adsorption sites on the MC surface and also lends a higher number of vacant sites where the chemical reaction between the metal and the amine compound can occur; both are important steps of the global adsorption mechanism. However, Figure 1 also shows that under some experimental conditions a certain degree of agglomeration of the microspheres is produced and also the adherence of some organic material on the surface of the microcapsules. This occurrence reflects the lack of arabic gum, the dispersion agent, which is used to prevent aggregation of the microcapsules. In all these experiments, the same amount of dispersant was used, which indicates that the quantity of this compound should be increased in proportion to increases in the amount of the extractant used in the polymerization.

Table 2 shows the adsorption and desorption pore diameters of microcapsules synthesized using different monomer compositions. The pore size decreases as the content of styrene in the polymer matrix increases. The surface area measured for the microspheres averages around $480 \mathrm{~m}^{2} \mathrm{~g}^{-1}$ and the pore volume around $0.40 \mathrm{~cm}^{3} \mathrm{~g}^{-1}$, which decreases slightly for microcapsules with a higher content of styrene in their solid polymeric structure. 
Table 2: Pore size of microcapsules determined by porosimetry.

\begin{tabular}{|l|c|c|c|}
\hline \multicolumn{1}{|c|}{ Pore size [nm] } & $\mathrm{MC}_{20 / 80}$ & $\mathrm{MC}_{40 / 60}$ & $\mathrm{MC}_{60 / 40}$ \\
\hline $\begin{array}{l}\text { BJH adsorption average pore } \\
\text { diameter (2V/A) }\end{array}$ & 1.7668 & 1.6075 & 0.4932 \\
\hline $\begin{array}{l}\text { BJH desorption average pore } \\
\text { diameter (2V/A) }\end{array}$ & 1.6026 & 1.5106 & 1.4780 \\
\hline
\end{tabular}

\section{BJH: Barret-Joyner-Halenda calculation}

These results may be attributed to the influence of divinylbenzene on the polymeric matrix structure. When the fraction of cross-linker increases a solid matrix is generated whose structure contains many polymeric chains with a higher free-volume. In contrast, when microcapsules contain a higher fraction of styrene, the polymer chains may be produced that are too flexible, which generates a smaller free-volume. Pores are formed when the solvent is evaporated from the microcapsules as they are being formed. Therefore, evaporation of the solvent is affected by the hydrodynamic conditions in the reactor, including stirring speed, and most strongly by the characteristics and proportion of reactants and solvent used. Needless to say that these microspheres prepared without extractant did not present any chemical activity as metal- adsorbents.

Figure 2 shows a plot of the maximum particle size of the microcapsules as a function of the amount of amine extractant that was microencapsulated, expressed as its percent over the total organic mass used in the synthesis.

The distribution of particle size was very narrow in all cases, thus the maximum particle size practically correspond to the average size of microspheres. The size of the microcapsules varied from $42.8 \mu \mathrm{m}$ to 148.3 $\mu \mathrm{m}$. The figure shows that the maximum particle size decreases as the quantity of extractant immobilized in the polymeric matrix is increased. This result might be attributed to the higher content of extractant causing a decrease in the interfacial tension around the organics drops of the emulsion where the

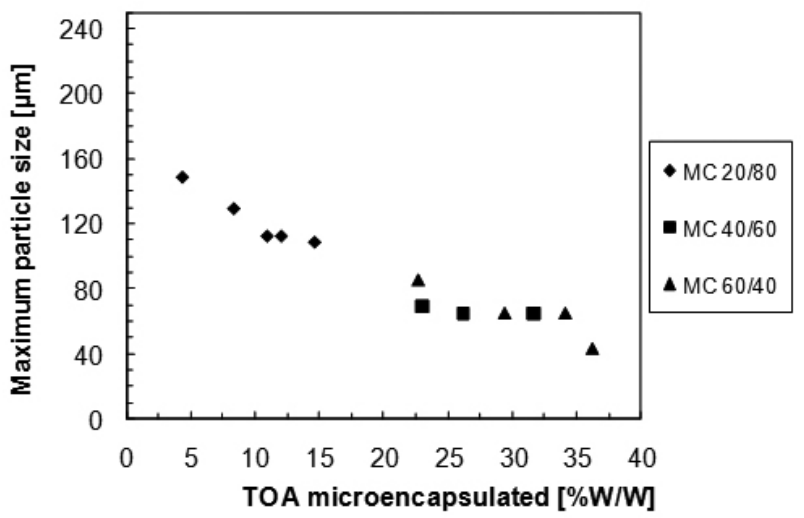

Figure 2: Dependence of microcapsule particle size on the amount of extractant used in the synthesis.

reaction of polymerization occurs. Thus, smaller drops are produced, which lead to the formation of smaller microcapsules. It is well known that these type of extractant compounds present high surface activity because of their polar head and their long-alkyl chain ${ }^{17}$. The head contains the amine-functional group, which makes it an active species with extraction ability, and its alkydic chain imparts its solubility in organic diluents. Another factor that affects size of the obtained microcapsules is the stirring speed during the polymerization reaction, however, this variable was kept constant in all experiments.

Table 3 presents the results of immobilized extractant as a function of the quantity of TOA used in the polymerization reaction. The quantity of immobilized amine depends on the porosity and tortuosity of the solid matrix. A smaller porosity implies an early saturation of the polymeric structure of the microcapsule, preventing a higher retention of the extractant in them by capillarity action. As was indicated above, as the amount of trioctylamine added to the reaction is increased, smaller organic drops are generated, leading to smaller microspheres, which results in a larger total surface area and allows for larger retention of the extractant.
Table 3: Dependence of immobilization of the extractant in the microcapsules on the amount of TOA used in the polymerization.

\begin{tabular}{|c|c|}
\hline $\mathrm{MC}_{\mathrm{ST/DVB}} \mathrm{TOA}$ & $\begin{array}{c}\text { TOA retained in MC } \\
{[\mathrm{mmoles} / \mathrm{g} \mathrm{MC}]}\end{array}$ \\
\hline $\mathrm{MC}_{20 / 80} 8.4$ & 0.0567 \\
\hline $\mathrm{MC}_{20 / 80} 11.0$ & 0.1574 \\
\hline $\mathrm{MC}_{20 / 80} 14.7$ & 0.3467 \\
\hline $\mathrm{MC}_{40 / 60} 19.6$ & 0.6178 \\
\hline $\mathrm{MC}_{40 / 60} 26.2$ & 0.7217 \\
\hline $\mathrm{MC}_{40 / 60} 31.7$ & 0.7799 \\
\hline $\mathrm{MC}_{60 / 40} 29.4$ & 0.8077 \\
\hline $\mathrm{MC}_{60 / 40} 34.1$ & 1.2939 \\
\hline $\mathrm{MC}_{60 / 40} 36.2$ & 1.7560 \\
\hline
\end{tabular}

\subsection{Extraction kinetics experiments}

One of the most interesting questions to ask, when adsorbents like these microcapsules are used for metal uptake from aqueous solutions, is at what rate adsorption is favored. Extraction kinetics tests were carried out by mixing the synthesized $\mathrm{MC}$ with aqueous solutions containing either zinc or copper ions whose initial $\mathrm{pH}$ was 2.0. Samples of the raffinate solutions were taken at time intervals and were then analyzed by atomic absorption spectrophotometry to measure the residual amount of metal present. The amount of metal adsorbed in the microcapsules was calculated from the mass balance between initial and final metal concentrations in the acid aqueous solution. In Figure 3 the results are shown for the uptake of $\mathrm{Zn}$ (II) ions from feed solutions using different microcapsules. It is observed that in all cases the extraction of metal from the acid solutions is achieved within a few minutes, confirming that this extractant, when immobilized in the microcapsules, maintains its fast kinetics to react with the metal as is normally observed in liquid-liquid extraction systems.

Figure 3 also shows that the microcapsules whose polymeric matrix retained a higher amount of the amine, which correspond to those synthesized with a higher quantity of extractant, presented a better metal loading capacity. This fact is consistent with the above argument that a higher amount of extractant used during preparation leads to the production of smaller-sized microcapsules with larger surface areas, which enhances the chemical reaction with the metal because there is a larger amount of extractant molecules available at

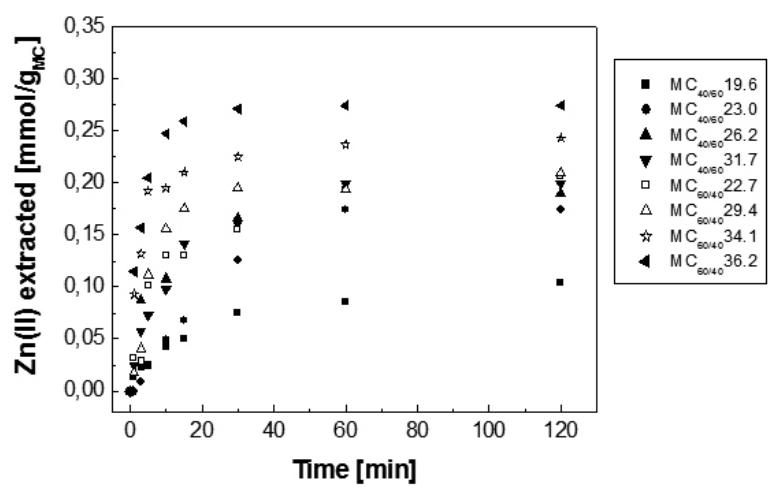

Figure 3: $\mathrm{Zn}(\mathrm{II})$ ions adsorption with TOA onto microcapsules.

the interface. In Figure 3 is also observed a somewhat higher metal loading with MC whose polymeric matrix consists of $60 \%$ of styrene and $40 \%$ of divinylbenzene compared to those that contain a higher proportion of DVB. A higher proportion of styrene also produces smaller microspheres which would benefit the occurrence of the chemisorption process due to the enhanced contact between the extractant retained in the porous of the solid matrix and the metallic ion contained in the aqueous feed solution.

In previous reports, we have remarked on the importance of the chemical reaction between the metal and the extractant in the overall process of 
chemisorption. It is well known that amine-type extractants act by ion-pair formation ${ }^{18,19}$. Trioctylamine contains a basic nitrogen atom capable of forming amine salts with a wide variety of inorganic and organic acids, which allow it to extract the metals following a mechanism based on the principle of ion association, whereby a large and positively charged organic species produces the extraction of a large and anionic metal complex toward the organic phase, which is in this case retained on the microcapsules. Equation (1) represents the amine salt formation, $\mathrm{R}_{3} \mathrm{NH}^{+} \mathrm{Cl}$, by reaction of the organic extractant with hydrochloric acid existent in the aqueous feed solution at the interface between the aqueous solution and the surface of MC. In turn, equations (2) and (3) represent true ion exchanges with the anionic species of both metals. These equations can be ascribed to the fact that under the chloride-concentration conditions used in the aqueous solution, $\mathrm{Zn}$ (II) and $\mathrm{Cu}(\mathrm{II})$ are capable of forming very stable anionic complexes $\mathrm{ZnCl}_{4}^{2-}$ and $\mathrm{CuCl}_{4}^{2-20}$.

Amine salt formation: $\mathrm{R}_{3} \mathrm{~N}_{\text {org }}+\mathrm{HCl}_{a q}=\mathrm{R}_{3} \mathrm{NH}^{+} \mathrm{Cl}_{M C}$ Anion-Exchange: $\mathrm{ZnCl}_{4}^{2-}{ }^{\circ}+2 \mathrm{R}_{3} \mathrm{NH}^{+} \mathrm{Cl}_{M C}=\left(\mathrm{R}_{3} \mathrm{NH}^{+}\right)_{2} \mathrm{ZnCl}_{4 \mathrm{CC}}+2 \mathrm{Cl}$ (1) Anion-Exchange: $\mathrm{CuCl}_{4}{ }^{2-}{ }_{a q}^{a q}+2 \mathrm{R}_{3} \mathrm{NH}^{+} \mathrm{Cl}^{-}{ }_{\mathrm{MC}}=\left(\mathrm{R}_{3} \mathrm{NH}^{+}\right)_{2} \mathrm{CuCl}_{4 \mathrm{MC}}+2 \mathrm{Cl}^{-}{ }_{a q}$

where $R_{3} N$ denotes the high-molecular-weight tertiary amine extractant and $\left(\mathrm{R}_{3} \mathrm{NH}^{+}\right)_{2} \mathrm{ZnCl}_{4}$ and $\left(\mathrm{R}_{3} \mathrm{NH}^{+}\right)_{2} \mathrm{CuCl}_{4}$ the chloride-metal complexes adsorbed onto the microcapsules. The subscripts "aq", "org" and " $M C$ " denote species in the aqueous phase, organic solution or adsorbed in the microcapsule, respectively.

With respect to the kinetics behavior of different solid adsorbents, many works have been published concerning adsorption models to interpret the obtained results under determined experimental conditions ${ }^{21-26}$. The rate at which adsorption is enhanced depends on several variables, including the capacity of the adsorbent, the initial adsorbate concentration, the initial $\mathrm{pH}$ of the aqueous solution to treat, the particle size of adsorbent, the reaction temperature, and so on. In a previous paper, we performed a detailed revision of diverse kinetics adsorption models that are appropriate to apply when microcapsules are used as granular sorbents, describing the circumstances when those models could be applied to explain experimental results ${ }^{14}$. In the present study, the microcapsules were synthesized following a chemical method where the solvent is completely evaporated, causing the extractant be immobilized in the MC almost at a pure state. For this case, it possible to assume that a pseudosecond-order kinetics model can be applied to the experimental data, according to Sobkowsk and other authors ${ }^{27}$. This model is mainly based on the sorption capacity of the adsorbent which corresponds in this study to the microcapsules with the immobilized extractant, which is described by Ho, according to the following equation ${ }^{28}$ :

$$
\frac{d\left(q_{t}\right)}{d t}=k_{2}\left(q_{e}-q_{t}\right)^{2}
$$

where, $k_{2}$ is the rate constant of the pseudo-second-order expressed in $\mathrm{g}_{\mathrm{MC}} / \mathrm{mmol} \cdot \mathrm{sec}, q_{e}$ is the molar amount of metal ion adsorbed at equilibrium by gram of microcapsule $\left[\mathrm{mmol} / \mathrm{g}_{\mathrm{MC}}\right]$, and $q_{t}$ is the molarity of the metal adsorbed onto the $\mathrm{MC}$ at any time $t$ expressed per gram of microcapsule $\left[\mathrm{mmol} / \mathrm{g}_{\mathrm{MC}}\right]$. Equation (4) corresponds to an expression of the reaction between the metal and the extractant retained in the MC during the adsorption process, which is dependent of the free sorption active sites. Analogously, a similar equation can be used for the desorption process of the metal from the MC using suitable stripping agents; this stage of the study will be extensively reviewed in a later report.

Equation (4) was integrated with the boundary conditions of $t=0$ to $t=t$ and $q_{t}=0$ to $q_{t}=q_{t}$ to yield the following linear form:

$$
\frac{t}{q_{t}}=\frac{1}{k_{2} q_{e}^{2}}+\left(\frac{1}{q_{e}}\right) t
$$

where the product $k_{2} q_{e}^{2}$ represents the initial sorption rate, when $t$ tends to 0 , expressed in $\mathrm{mmol} / \mathrm{g}_{\mathrm{MC}}$. $\mathrm{sec}$. In Figure 4 the experimental data are plotted according to equation (5).

The pseudo-second-order rate constants $k_{2}$, the initial sorption rate $k_{2} q_{e}^{2}$ and the equilibrium sorption capacity were obtained from the plot by linear regression. In Table 4 the calculated values for the microcapsules $\mathrm{MC}_{60 / 40}$ and $\mathrm{MC}_{40 / 60}$ are listed, including the coefficient of determinations, R. All these experiments were carried out using an aqueous feed solution whose metal content was $100 \mathrm{mgL}^{-1}$ and $100 \mathrm{gL}^{-1}$ of $\mathrm{NaCl}$ at a temperature of $30^{\circ} \mathrm{C}$. These microcapsules were considered for the kinetics study since they showed a higher encapsulation of the extractant.

Table 4 shows that the highest rate constant for the extraction of zinc and the highest amount of metal ion adsorbed at equilibrium was obtained for the microcapsule $\mathrm{MC}_{60 / 40} 36.2$, which confirms the positive effect of using a higher quantity of extractant during the synthesis of microspheres, whose matrix is composed of an appropriate copolymer of styrene and divinylbenzene. In Table 4 it is also shown that good agreement was obtained between the experimentally observed values of $q_{e \text { exp }}$ and those calculated by using the pseudo-second-order kinetics model, $q_{e}$.

The same analysis was performed with the experimental results observed for the $\mathrm{Cu}(\mathrm{II})$ adsorption. Figure 5 shows the adsorption of this metal using different microcapsules loaded with the extractant trioctylamine.

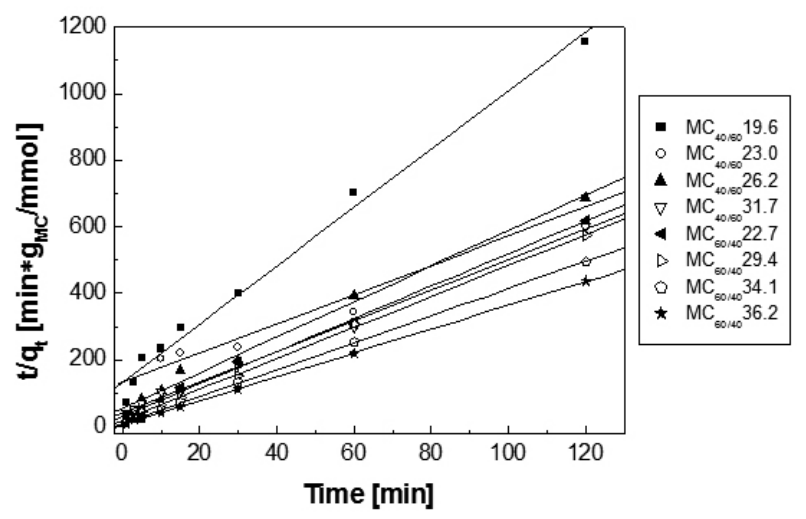

Figure 4: Pseudo-second-order kinetic model for the adsorption of $\mathrm{Zn}(\mathrm{II})$ onto microcapsules loaded with TOA.

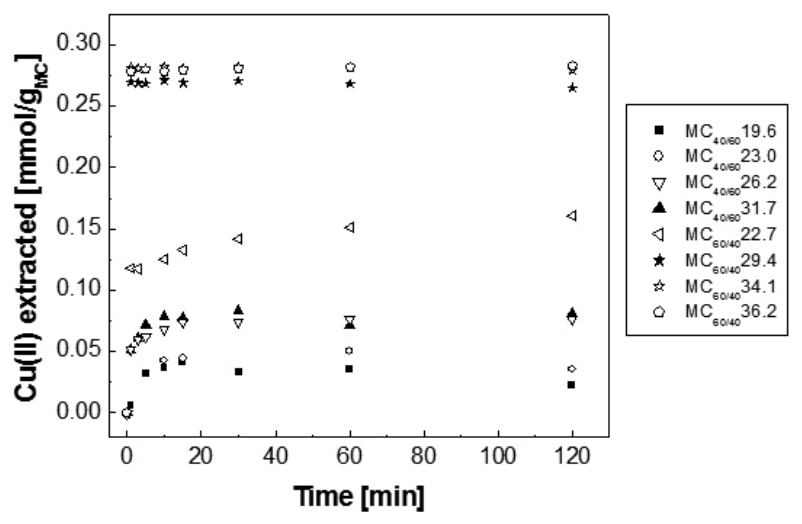

Figure 5: $\mathrm{Cu}(\mathrm{II})$ ions adsorption with TOA onto microcapsules.

A similar $\mathrm{Cu}$ (II) sorption behavior from aqueous solutions onto the polymeric microcapsules was seen with respect to that observed for $\mathrm{Zn}(\mathrm{II})$ i.e., the extraction is achieved within a few minutes and reaches a variable metal-loading capacity for each type of microsphere according its structure and content of active extractant. The fast kinetics of the sorptive chemical reaction that the metal undergoes with the extractant that is retained in the MC is finally governed by a liquid-liquid reaction, which normally proceeds faster than solid-liquid reactions like produced when using IX solid resins. Figure 6 shows the arrangement of experimental data measured for $\mathrm{Cu}(\mathrm{II})$ sorption onto the microcapsules according the linear form of the pseudo-second-order kinetics model expressed in equation (5).

It is clearly observed that in general all the data fit the kinetics model fairly well that was proposed based on the sorption capacity of the solid-sorbent. Other kinetics models applied to explain the experimental data (as first order or half order) did not fit the achieved results. In Table 5 the sorption parameters for this metal are presented as calculated from Figure 6. 


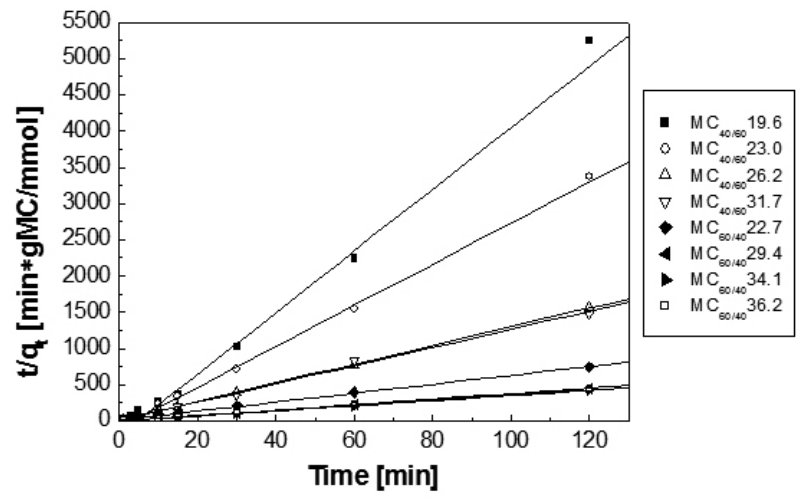

Figure 6: Pseudo-second-order kinetic model for the adsorption of $\mathrm{Cu}(\mathrm{II})$ with TOA immobilized onto polymeric microcapsules.

Table 4: Kinetic parameters of the pseudo-second-order model applied to zinc(II) adsorption onto MC loaded with TOA.

\begin{tabular}{|c|c|c|c|c|c|}
\hline $\mathrm{MC}_{\mathrm{St} / \mathrm{DVB}} \mathrm{TOA}_{\mathrm{ret}}$ & $\begin{array}{c}q_{e} \\
{\left[\mathrm{mmol} / \mathrm{g}_{\mathrm{MC}}\right]}\end{array}$ & $\begin{array}{c}k_{2} \\
{\left[\mathrm{~g}_{\mathrm{MC}} / \mathrm{mmol} \cdot \mathrm{sec}\right]}\end{array}$ & $k_{2} q_{e}^{2}\left[\mathrm{mmol} / \mathrm{g}_{\mathrm{MC}} \cdot \mathrm{sec}\right]$ & $\begin{array}{c}q_{e} \exp \\
{\left[\mathrm{mmol}_{\mathrm{g}}\right]}\end{array}$ & 0.1038 \\
\hline $\mathrm{MC}_{40 / 60} 19.6$ & 0.1141 & 0.5795 & 0.0075 & 0.1746 & 0.9930 \\
\hline $\mathrm{MC}_{40 / 60} 23.0$ & 0.2279 & 0.1449 & 0.0075 & 0.1905 & 0.9957 \\
\hline $\mathrm{MC}_{40 / 60} 26.2$ & 0.1872 & 0.5203 & 0.0182 & 0.1998 & 0.9986 \\
\hline $\mathrm{MC}_{40 / 60} 31.7$ & 0.2173 & 0.5100 & 0.0241 & 0.2064 & 0.9968 \\
\hline $\mathrm{MC}_{60 / 40} 22.7$ & 0.2049 & 0.7633 & 0.0320 & 0.2096 & 0.9996 \\
\hline $\mathrm{MC}_{60 / 40} 29.4$ & 0.2153 & 1.1327 & 0.0525 & 0.2430 & 0.9999 \\
\hline $\mathrm{MC}_{60 / 40} 34.1$ & 0.2471 & 1.7487 & 0.1067 & 0.2743 & 0.9999 \\
\hline $\mathrm{MC}_{60 / 40} 36.2$ & 0.2786 & 2.4791 & 0.1924 & & \\
\hline
\end{tabular}

Table 5: Kinetic parameters for sorption of $\mathrm{Cu}(\mathrm{II})$ on $\mathrm{MC}$ calculated by the pseudo-second-order model

\begin{tabular}{|c|c|c|c|c|c|}
\hline $\mathrm{MC}_{\mathrm{StDVB}} \mathrm{TOA}_{\mathrm{ret}}$ & $\begin{array}{c}\mathrm{q}_{\mathrm{e}} \\
{\left[\mathrm{mmol} / \mathrm{g}_{\mathrm{MC}}\right]}\end{array}$ & $\begin{array}{c}k_{2} \\
{\left[\mathrm{~g}_{\mathrm{MC}} / \mathrm{mmol} \cdot \mathrm{sec}\right]}\end{array}$ & $k_{2} q_{e}^{2}\left[\mathrm{mmol} / \mathrm{g}_{\mathrm{MC}} \cdot \mathrm{sec}\right]$ & $\begin{array}{c}\mathrm{q}_{\mathrm{e}} \exp \\
{\left[\mathrm{mmol} / \mathrm{g}_{\mathrm{MC}}\right]}\end{array}$ & $\mathrm{R}^{2}$ \\
\hline $\mathrm{MC}_{40 / 60} 19.6$ & 0.0235 & 8.869 & 0.0049 & 0.0229 & 0.9851 \\
\hline $\mathrm{MC}_{40 / 60} 23.0$ & 0.0353 & 8.066 & 0.0101 & 0.0360 & 0.9928 \\
\hline $\mathrm{MC}_{40 / 60} 26.2$ & 0.0776 & 13.345 & 0.0803 & 0.0769 & 0.9908 \\
\hline $\mathrm{MC}_{40 / 60} 31.7$ & 0.0797 & 15.764 & 0.1000 & 0.0806 & 0.9982 \\
\hline $\mathrm{MC}_{60 / 40} 22.7$ & 0.1622 & 2.469 & 0.0650 & 0.1608 & 0.9993 \\
\hline $\mathrm{MC}_{60 / 40} 29.4$ & 0.2648 & 16.801 & 2.1783 & 0.2646 & 0.9999 \\
\hline $\mathrm{MC}_{60 / 40} 34.1$ & 0.2794 & 34.868 & 2.4507 & 0.2791 & 0.9999 \\
\hline $\mathrm{MC}_{60 / 40} 36.2$ & 0.2830 & 30.596 & & 0.2830 & 1.0000 \\
\hline
\end{tabular}

Microcapsules prepared using $60 \%$ of styrene and $40 \%$ of divinylbenzene and retaining a high amount of the amine extractant presented a better $\mathrm{Cu}(\mathrm{II})$ sorption behavior, which corroborates the results reached for zinc adsorption. Table 5 illustrates good agreement with the proposed kinetics model, given that the linear regression coefficient of measurements were all extremely high.

Sorption extents close to $90 \%$ under optimal experimental conditions were reached for both metals with respect to the initial content of zinc and copper in the aqueous feed solutions. These results also indicate that the rate of $\mathrm{Zn}(\mathrm{II})$ and $\mathrm{Cu}(\mathrm{II})$ sorption processes would be effectively controlled by the chemical process described by the pseudo-second-order reaction mechanism proposed in this study. These polymeric matrices were found be appropriate for retaining the extractant and no leakage of the organic compounds to the aqueous solutions was observed. Moreover, the results confirm that trioctylamine is a suitable extractant for metals forming anionic-species in aqueous solutions, following the chemical reactions indicated in equations $1-3$, which is applicable to situations that occur in many industrial cases as was described above. The chlorine-complexes of both metals, $\mathrm{ZnCl}_{4}{ }^{2-}$ and $\mathrm{CuCl}_{4}{ }^{2-}$, are quite stable in aqueous solutions under the experimental conditions used in this study and their complex-compounds formed with the extractant remained efficiently immobilized inside the porous structure of the microspheres synthesized in this work. Kinetics information obtained herein is valuable for understanding the principles that govern the chemisorption process, which must be applied in designing a practical process grounded in the use of continuous columns packed with microcapsules. It is clear that these microcapsules can be used in industrial wastewater treatment, behaving as ion-exchanger, to replace undesirable heavy metal ions like copper and zinc with other, less hazardous contaminants. Microcapsule methodology is technologically simple and cheap, which makes it especially suitable for treating dilute polluted waters. It can be applied to remove all ions present in aqueous solutions if an appropriate extractant is retained onto the microcapsules and applied under favorable conditions, like the use of TOA for the uptake of zinc and copper at $\mathrm{pH}$ 2.0. If the purpose were to separate some of the metals existent in the feed solution, a selective removal would also be possible by selecting the suitable extractant and applying it following determined experimental conditions. The decision 
of a collective removal of all ions or the selective separation of some of them would depend on the solution to treat or on the grade of treatment required.

\section{CONCLUSIONS}

The microencapsulation of the non-specific extractant trioctylamine in polymeric matrices was studied for their application in the removal of $\mathrm{Zn}(\mathrm{II})$ and $\mathrm{Cu}(\mathrm{II})$ from aqueous solutions. Microcapsules were prepared using an in situ free radical suspension polymerization method using styrene and divinylbenzene as monomers. Diverse microcapsules were prepared using different proportions of monomers and a variable amount of extractant. It was found by SEM analysis that the microcapsules have spherical shapes and particle sizes varying from $42.8 \mu \mathrm{m}$ to $148.3 \mu \mathrm{m}$. Porosimetry analysis indicated that the microcapsules present an average surface area of $480 \mathrm{~m}^{2} \mathrm{~g}^{-1}$, a pore volume of $0.40 \mathrm{~cm}^{3} \mathrm{~g}^{-1}$ and a pore size averaging a value of $1.6 \mathrm{~nm}$.

With respect to the synthesis of the microcapsules, the higher the amount of extractant used during their preparation the smaller the yield of the process, probably due to the inhibitory effect of an excess of amine on the polymerization reaction, and also causes an increase in the viscosity of the reaction medium affecting the yield too. However, in these conditions smaller microcapsules are produced, which present a larger surface area favoring the encapsulation of the amine. Concerning the polymerization reaction, a higher proportion of divinylbenzene favored the synthesis of microspheres, however, the presence of styrene assured a suitable wettability of the microcapsules by the feed solution, enhancing the metal adsorption process.

$\mathrm{Zn}(\mathrm{II})$ and $\mathrm{Cu}(\mathrm{II})$ sorption kinetics experiments from aqueous solutions were carried out using microcapsules loaded with the amine-type extractant Results show that the extraction of metals is achieved within a few minutes following the same chemical mechanism observed in liquid-liquid extractions, where the alkylamine interacts by ion-pair formation with the metal species present in aqueous solutions through an anion-exchange reaction onto the surface of the microcapsules. Microcapsules retaining a higher amount of extractant were constituted of $60 \%$ of styrene and $40 \%$ of DVB and presented a better and faster metal-loading capacity, coherently with the above explanations.

A pseudo-second-order rate kinetics model was applied to explain the experimental results of the chemisorption of both metals onto the microcapsules containing trioctylamine. The kinetics model fit the obtained results well, confirming that the sorption of metals onto the microspheres was based predominantly on the sorption capacity of the solid sorbent Microcapsules behave as granular sorbents immobilizing the active extractant in their structure, practically at a pure state after solvent evaporation. A good agreement was found between the observed values of experimentally adsorbed amount of metals, $q_{e}$, with those calculated by the pseudo-second-order kinetics model. A higher rate constant, $k_{2}$ and a higher initial sorption rate was determined for copper respect to zinc. However, under favorable experimental conditions, adsorption extents around $90 \%$ were measured for both metals, confirming that trioctylamine is a suitable extractant for metals forming chlorine-complexes in aqueous solutions. We can conclude from the results of this study that these types of microcapsules would provide a promising alternative for treating residual aqueous solutions polluted with heavy metal ions. Their synthesis is simple and cheap and they present a strong metalsorption capacity. Depending of the subsequent use of the treated water by this methodology, it could be necessary a later step of neutralization prior to their discharge into water bodies according to environmental regulations.

\section{ACKNOWLEDGEMENTS}

This work was supported by a research grant from The Fondo Nacional de Ciencia y Tecnología, Chile (FONDECYT) N N $^{\circ} 1070608$. C. Fonseca and C. Araneda acknowledge Doctoral Fellowship from CONICYT-Chile and the research grants received from The University of Chile and The School of Chemical and Physical Sciences of The Victoria University of Wellington.

\section{REFERENCES}

1. K. Saji-John, J. Saji, M. Reddy, T. Ramamohan, T. Rao, Hydrometallurgy 51, 9, (1999).

2. J. Dutrizac, Hydrometallurgy 29, 1, (1992).

3. L. Lin, R. Juang, Chem. Eng. J. 112, 211, (2005).

4. M. Matlock, B. Howerton, D. Atwood, Water Res. 36, 4757, (2002).

5. F. Valenzuela, J. Andrade, J. Sapag, C. Tapia, C. Basualto, Miner. Eng. 8, 893, (1995).

6. J. Xu, L. Wang, W. Shen, R. Paimin, X. Wang, Sep. Sci. Technol. 39, $3527,(2004)$.

7. W. Yang, G. Luo, X. Gong, Sep. Purif. Technol. 43, 175, (2005).

8. N. Ochoa, C. Illanes, J. Marchese, C. Basualto, F. Valenzuela, Sep. Purif. Technol. 52, 39, (2006).

9. F. Valenzuela, M. Yazdani, C. Araneda, C. Basualto, E. Kamio, K. Kondo, J. Chil. Chem. Soc. 50, 711, (2005).

10. E. Kamio, Y. Fujiwara, M. Matsumoto, F. Valenzuela, K. Kondo, Chem. Eng. J. 139, 93, (2008).

11. E. Kamio, M. Matsumoto, F. Valenzuela, K. Kondo, Ind. Eng. Chem. Res. 44, 2266, (2005).

12. S. Nishihama, G. Nishimura, T. Hirai, I. Komasawa, Ind. Eng. Chem. Res. 43, 751, (2004).

13. K. Shiomori, H. Yoshizawa, K. Fujikubo, Y. Kawano, Y. Hatate, Y. Kitamura, Sep. Sci. Technol. 38, 4057, (2003).

14. C. Araneda, C. Fonseca, J. Sapag, C. Basualto, M. Yazdani, K. Kondo, E. Kamio, F. Valenzuela, Sep. Purif. Technol. 63, 517, (2008).

15. G. Barassi, A. Valdés, C. Araneda, C. Basualto, J. Sapag, C. Tapia, F. Valenzuela, J. Hazard, Mater. 172, 262, (2009).

16. I. Mishonov, G. Kyuchoukov, Hydrometallurgy 41, 89, (1996).

17. F. Valenzuela, C. Salinas, C. Basualto, J. Sapag, C. Tapia, J. Chil. Chem. Soc. 48, 79, (2003).

18. C. Basualto, J. Marchese, F. Valenzuela, A. Acosta, Talanta 59, 999, (2003).

19. J. Marchese, F. Valenzuela, C. Basualto, A. Acosta, Hydrometallurgy 72, 309, (2004).

20. O. Parchment, M. Vincent, I. Hillier, J. Phys. Chem. 100, 9689, (1996).

21. Y. Ho, G. McKay, Water Res. 34, 735, (2000).

22. Y. Ho, C. Wang, Process Biochem. 39, 759, (2004).

23. S. Azizian, J. Colloid. Interf. Sci. 276, 47, (2004).

24. Y. Zhang, J. Chen, X. Yan, Q. Feng, J. Chem. Thermodyn. 39, 862, (2007).

25. Y. Sharma, C. Weng, J. Hazard. Mater. 142, 449, (2007).

26. E. Demirbas, N. Dizge, M. Sulak, M. Kobya, Chem. Eng. J. 148, 480, (2009).

27. J. Sobkowsk, A. Czerwinski, J. Electroanal. Chem. 55, 391, (1974).

28. Y. Ho, J. Hazard. Mater. 136, 681, (2006). 